\title{
ANÁLISE FATORIAL DO MASLACH BURNOUT INVENTORY (MBI) EM UMA AMOSTRA DE PROFESSORES DE INSTITUIÇÕES PARTICULARES
}

\author{
Mary Sandra Carlotto \\ Sheila Gonçalves Câmara"
}

\begin{abstract}
RESUMO. O Inventário de Burnout de Maslach (MBI) foi projetado para avaliar a Síndrome de Burnout em trabalhadores. Este artigo analisa o comportamento psicométrico (fidedignidade e validade de construto) do MBI numa amostra de 563 professores que atuam em instituições particulares de ensino fundamental, médio e superior, na região metropolitana de Porto Alegre-RS. A análise fatorial identificou três fatores correlatos às três dimensões originalmente identificadas pelo inventário: exaustão emocional, despersonalização e realização profissional. Os três fatores explicaram, neste estudo, $47 \%$ da variância total das respostas dos sujeitos ao MBI. Todos eles alcançaram um nível satisfatório de consistência interna, sendo que o fator 1 (exaustão emocional) e o fator 2 (realização profissional) poderiam funcionar independentemente. Já o fator 3 (despersonalização) é utilizado no âmbito deste estudo em nível explicativo, contribuindo para uma compreensão mais ampla acerca da configuração de Burnout em professores.
\end{abstract}

Palavras-chave: Burnout, Inventário de Burnout de Maslach (MBI), análise fatorial.

\section{FACTORIAL ANALYSIS OF THE MASLACH BURNOUT INVENTORY (MBI) IN A SAMPLE OF TEACHERS FROM PRIVATE SCHOOLS}

\begin{abstract}
The Maslach Burnout Inventory (MBI) was projected to evaluate the Burnout Syndrome in working people. This article analyses the psychometric behaviour (reliability and construct validity) of the MBI in a sample of 563 teachers, workers from private institutions of elementary, high school and college levels in the metropolitan area of Porto Alegre-RS. The factorial analysis identified three factors originally related to the three dimensions identified in the inventory: emotional exhaustion, depersonalization and professional accomplishment. The three factors explained in this study $47 \%$ of the total variance of the answers by the individuals subjected to MBI. All of them reached a satisfactory level of consistence, and the factor 1 (emotional exhaustion) and the factor 2 (professional accomplishment) could function independently. As for the factor 3 (depersonalization), in the present study it is used in an explanatory level, contributing to a wider understanding concerning the configuration of Burnout in teachers.
\end{abstract}

Key words: Burnout, the Maslach Burnout Inventory (MBI), factorial analysis.

O presente trabalho constitui-se de um estudo complementar que faz parte de uma pesquisa mais ampla, na qual visamos identificar a associação da síndrome de burnout (SB) com variáveis demográficas e profissionais características de cargo e satisfação laboral (Carlotto, 2002 e 2003). Nosso interesse neste estudo centrou-se em uma avaliação do Maslach Burnout Inventory (MBI), de maneira a identificar sua distribuição fatorial em nosso contexto.

O termo burnout foi utilizado primeiramente por Freudenberger, médico psicanalista que descreveu este fenômeno como um sentimento de fracasso e exaustão causado por um excessivo desgaste de energia e recursos. Freudenberger complementou seus estudos em 1975 e 1977, incluindo em sua definição comportamentos de fadiga, depressão, irritabilidade, aborrecimento, sobrecarga de trabalho, rigidez e inflexibilidade (Freudenberger, 1974; França, 1987; Perlman \& Hartman, 1982).

As primeiras pesquisas sobre a SB são resultado de um trabalho sobre o estudo das emoções e maneiras de lidar com elas, desenvolvido com profissionais que, pela natureza de seu trabalho, necessitavam manter contato direto com outras pessoas (trabalhadores da

\footnotetext{
* Psicóloga. Especialista em Gestão de Recursos Humanos (UCAM-RJ), Mestre em Saúde Coletiva (ULBRA-RS), Docente da área de Psicologia do Trabalho do Curso de Psicologia - ULBRA/Canoas.

\# Psicóloga. Mestre em Psicologia Social e da Personalidade e Doutora em Psicologia (PUCRS), Docente da área de Psicologia Social Comunitária do Curso de Psicologia - ULBRA/Canoas.
} 
área da saúde, serviços sociais e educação), uma vez que se percebia a manifestação de estresse emocional e sintomas físicos por parte de tais profissionais. Os estudos iniciais foram realizados a partir de experiências pessoais de alguns autores, estudos de caso, estudos exploratórios, observações, entrevistas ou narrativas baseadas em programas e populações específicos (Cordes \& Dougherty, 1993; Maslach, Schaufeli, \& Leiter, 2001). Dos trabalhos publicados sobre SB entre 1974 e 1981, segundo Perlman e Hartman (1982), apenas cinco, identificados neste período, tratavam do fenômeno com alguma evidência empírica.

Assim, verifica-se que somente a partir de 1976 os estudos sobre burnout adquiriram um caráter científico, uma vez que foram construídos modelos teóricos e instrumentos capazes de registrar e compreender este sentimento crônico de desânimo, apatia e despersonalização. Christina Maslach, psicóloga social, foi quem entendeu primeiramente, em estudos com profissionais de serviços sociais e de saúde, que as pessoas com burnout apresentavam atitudes negativas e de distanciamento pessoal. Christina Maslach, Ayala Pines e Cary Cherniss foram os estudiosos que popularizaram o conceito de burnout e o legitimaram como uma importante questão social (Farber, 1991).

A definição mais aceita atualmente sobre a SB fundamenta-se na perspectiva social-psicológica (Maslach \& Jackson, 1981; Maslach \& Leiter, 1997; Maslach \& Golberg, 1998). Esta perspectiva considera a síndrome como uma reação à tensão emocional crônica causada por se lidar excessivamente com pessoas. É um construto formado por três dimensões relacionadas, mas independentes. A primeira delas é a de exaustão emocional, caracterizada pela falta ou carência de energia e entusiasmo e sentimento de esgotamento de recursos. È possível somarem-se sentimentos de frustração e tensão, pois os trabalhadores podem perceber que já não têm condições de despender mais energia para o atendimento de seu cliente ou demais pessoas, como faziam antes. Outra dimensão é a de despersonalização, situação em que o profissional passa a tratar os clientes, colegas e a organização como objetos. Os trabalhadores podem desenvolver uma insensibilidade emocional. A terceira dimensão é e baixa realização pessoal no trabalho, definida como uma tendência do trabalhador a se auto-avaliar de forma negativa. As pessoas se sentem infelizes consigo mesmas e insatisfeitas com seu desenvolvimento profissional. Também experimentam um declínio do sentimento de competência e êxito, bem como de sua capacidade de interagir com os outros.

De acordo com Maslach, Schaufeli e Leiter (2001), o que tem emergido de quase todos os estudos é a conceituação de burnout como uma síndrome psicossocial surgida como uma resposta crônica aos estressores interpessoais ocorridos na situação de trabalho. Depois de anos de investigações mantém-se a consistência dos fatores situacionais inicialmente correlacionados a este fenômeno. "Os resultados destes estudos têm deixado claro o impacto da situação de trabalho sobre o Burnout individual" (p. 401).

Os avanços nos estudos da SB têm ocorrido, porquanto as questões metodológicas têm sido qualificadas desde a sua fase pioneira. Schaufeli, Maslach e Marek (1993) observam que os progressos localizam-se em três grandes áreas: as medidas adotadas, uma vez que o MBI tem sido o instrumento utilizado pela maioria dos pesquisadores; os estudos transnacionais; e as investigações sobre o processo de desenvolvimento da síndrome.

\section{HISTÓRICO E EVOLUÇÃO DO MBI}

O instrumento mais utilizado para avaliar burnout, independentemente das características ocupacionais da amostra e de sua origem, segundo Gil-Monte e Peiró (1999), é o MBI - Maslach Burnout Inventory, elaborado por Christina Maslach e Susan Jackson em 1978. Sua construção partiu de duas dimensões, exaustão emocional e despersonalização, sendo que a terceira dimensão, realização profissional, surgiu após estudo desenvolvido com centenas de pessoas de uma ampla gama de profissionais (Maslach, 1993).

Inicialmente, o inventário possuía 47 itens que foram administrados em uma amostra de 605 sujeitos de várias ocupações profissionais. Dez fatores emergiram e, por meio de uma avaliação criteriosa, foram eliminados seis deles, juntamente com 24 itens que não possuíam peso fatorial superior a 0,40. Após aplicação em uma nova amostra de 420 sujeitos com perfil igual ao anterior, os mesmos quatro fatores emergiram, sendo que somente três destes apresentaram significância empírica (Maslach \& Jackson, 1981; Cordes \& Dougherty, 1993; Moreno, Bustos, Matallana \& Mirrales, 1997). A consistência interna das três dimensões do inventário é satisfatória, pois apresenta um alfa de Cronbach que vai desde 0,71 até 0,90 e os coeficientes de teste e reteste vão de 0,60 a 0,80 em períodos de até um mês (Maslach \& Jackson, 1981). 
O MBI avalia como o trabalhador vivencia seu trabalho, de acordo com três dimensões conceituais: exaustão emocional, realização profissional e despersonalização. Em sua primeira versão, o inventário avaliava a intensidade e a frequiência das respostas com uma escala de pontuação do tipo Likert, variando de 0 a 6 (Maslach \& Jackson, 1981; Maslach \& Leiter, 1997). A segunda edição do MBI, realizada em 1986, passou a utilizar somente a avaliação da freqüência, pois foi detectada a existência de alta associação entre as duas escalas, sendo que muitos estudos apontaram correlação superior a 0,80 (Maslach \& Jackson, 1986; Maslach, 1993; Moreno e cols., 1997).

O MBI é um instrumento utilizado exclusivamente para a avaliação da síndrome, não levando em consideração os elementos antecedentes e as consequiências de seu processo. Ele avalia índices de burnout de acordo com os escores de cada dimensão, sendo que altos escores em exaustão emocional e despersonalização e baixos escores em realização profissional (esta subescala é inversa) indicam alto nível de burnout (Maslach \& Jackson, 1986). Gil-Monte e Peiró (1997) reforçam a importância de avaliar o MBI como um construto tridimensional, ou seja, as três dimensões devem ser avaliadas e consideradas, a fim de manter sua perspectiva de síndrome.

Diversos estudos de validação do MBI têm apresentado diferentes distribuições fatoriais, normalmente variando de 3 a 5 fatores. Neste sentido, este estudo objetivou explorar a distribuição do MBI na população de professores de instituições privadas de ensino da região metropolitana de Porto Alegre-RS.

\section{MÉTODO}

\section{Participantes}

A amostra se constituiu de 563 professores que exercem atividade docente em instituições particulares de ensino localizadas na região metropolitana de Porto Alegre-RS. O estudo foi realizado em oito escolas e uma universidade. A maior parte do grupo pertence ao sexo feminino $(69,4 \%)$, é casada $(62 \%)$, possui filhos $(58,1 \%)$ e concentra-se na faixa de 30 a 50 anos $(68,9 \%)$. Os professores trabalham de 20 a 40 horas semanais $(80,5 \%)$ e possuem, em média, 12 anos e 6 meses $(\mathrm{dp}=9,25)$ de experiência profissional. Do grupo estudado, $50,08 \%$ pertencem ao ensino superior e $49,92 \%$ aos ensinos infantil, médio e fundamental.

\section{Instrumento}

O MBI foi incluído em um instrumento mais amplo, composto de cinco áreas referentes a dados demográficos, profissionais, características de cargo e satisfação laboral e Síndrome de burnout (MBI) de Maslach e Jackson (1986). Neste estudo, somente abordaremos os dados referentes a este último.

O inventário, traduzido e adaptado por Lautert (1995), é auto-aplicado e totaliza 22 itens. Em sua versão americana, a freqüência das respostas é avaliada através de uma escala de pontuação que varia de 0 a 6 . Utilizamos, neste estudo, o sistema de pontuação de 1 a 5, também usado por Tamayo (1997) na adaptação brasileira do instrumento, pois foi verificado que os sujeitos apresentavam dificuldade em responder muitos itens dos instrumentos, devido à especificidade dos critérios da escala original. Embora tenhamos optado pela escala de 5 itens, utilizamos o mesmo tipo de categorias de freqüência utilizadas na versão americana (Maslach \& Jackson,1986). Isto é, 1 para nunca, 2 para algumas vezes ao ano, 3 para algumas vezes ao mês, 4 para indicar algumas vezes na semana e 5 para diariamente. Na versão original americana, a consistência interna das três dimensões do inventário é satisfatória, pois apresenta um alfa de Cronbach que vai de 0,71 até 0,90 e os coeficientes de teste e reteste vão de 0,60 a 0,80 em períodos de até um mês (Maslach \& Jackson, 1981).

\section{Procedimentos}

Para a coleta dos dados, primeiramente foi realizado um contato com as direções das instituições de ensino, quando foi apresentado o objetivo do estudo a fim de obter a autorização e o apoio para a aplicação do instrumento. Este foi entregue aos professores em seus diários de classe, tendo sido a coleta realizada utilizando-se urnas colocadas no apoio docente ou na sala de professores. A pesquisa tem aprovação do Comitê de Ética da instituição de afiliação dos pesquisadores, tendo sido realizados os procedimentos éticos conforme resolução 196 do Conselho Nacional de Saúde (CNS), no que diz respeito à pesquisa com seres humanos (Hutz \& Spink, 1996). O banco de dados foi digitado e, posteriormente, analisado no pacote estatístico SPSS, versão 10.0 .

A validação das dimensões de burnout foi feita através de medidas de adequação da amostra à análise fatorial, determinação do número de fatores adequados à análise fatorial, validade de construto pelo método de componentes principais, confiabilidade pelo método do alfa de Cronbach e normatização utilizando-se estatísticas descritivas dos construtos resultantes. 


\section{RESULTADOS}

\section{Validação do construto}

Antes da realização da análise fatorial foram contemplados alguns critérios, necessários à sua realização. A adequação da amostra foi mensurada pelos seguintes critérios: determinante da matriz de correlação, medida de adequação da amostra de Kaiser-Meyer-Olkin (KMO), teste de esfericidade de Bartlett e medidas individuais de adequação da amostra (MSA).

$\mathrm{O}$ valor do determinante da matriz de correlação calculado para a amostra foi de 6,74E-4, um valor bastante baixo, indicando que as variáveis são bastante intercorrelacionadas. Esta intercorrelação foi confirmada pelo teste de esfericidade de Bartlett $\left(X^{2}=\right.$ $3722,9 ; \mathrm{p}=0,000)$. Tais valores rejeitam a hipótese nula de que não haja intercorrelação entre os valores. Ao contrário, existem intercorrelações estatisticamente significativas e de valores altos. Isso indica que a matriz de dados é adequada para proceder à análise fatorial. $\mathrm{O}$ índice de adequação da amostra de KMO foi calculado em 0,904, considerado excelente por Bisquerra-Alzina (1989). Os índices de MSA individuais foram todos altos, variando de 0,773 a 0,938 .

Para realização da análise fatorial incluímos os 22 itens do MBI, que se encontram descritos em sua distribuição fatorial na matriz estrutural (Tabela 1). Assumindo-se, de acordo com o manual do MBI, que os fatores são independentes entre si (Maslach, Jackson \& Leiter, 1996), foi utilizado o método de extração de fatores usando componentes principais, com rotação ortogonal Varimax. Os três fatores encontrados a partir da distribuição de seus componentes e de seus autovalores (eigenvalue $\geq$ 1,000) nos permitiram identificar uma distribuição fatorial bem delimitada tanto em termos de proximidade dos itens na análise quanto em termos de aderência à teoria.

Tabela 1. Matriz estrutural das dimensões de Burnout.

\begin{tabular}{llcc}
\hline Itens & \multicolumn{3}{c}{ Dimensões } \\
\cline { 2 - 5 } & EE & RP & DE \\
\hline MBI 08 & $\mathbf{0 , 7 9}$ & 0,19 & 0,10 \\
MBI 02 & $\mathbf{0 , 7 5}$ & 0,16 & 0,08 \\
MBI 03 & $\mathbf{0 , 7 4}$ & 0,21 & 0,03 \\
MBI 14 & $\mathbf{0 , 7 3}$ & 0,02 & 0,08 \\
MBI 13 & $\mathbf{0 , 6 6}$ & 0,24 & 0,24 \\
MBI 20 & $\mathbf{0 , 6 5}$ & 0,11 & 0,10 \\
MBI 01 & $\mathbf{0 , 6 0}$ & 0,25 & 0,09 \\
MBI 06 & $\mathbf{0 , 5 9}$ & 0,21 & 0,19 \\
MBI 16 & $\mathbf{0 , 5 8}$ & 0,09 & 0,26 \\
MBI 09 & 0,04 & $\mathbf{0 , 7 2}$ & 0,04 \\
MBI 19 & 0,26 & $\mathbf{0 , 6 9}$ & 0,11 \\
MBI 17 & 0,17 & $\mathbf{0 , 6 8}$ & 0,15 \\
MBI 21 & 0,06 & $\mathbf{0 , 6 5}$ & 0,07 \\
MBI 18 & 0,22 & $\mathbf{0 , 6 5}$ & 0,11 \\
MBI 12 & 0,33 & $\mathbf{0 , 6 4}$ & 0,06 \\
MBI 07 & 0,06 & $\mathbf{0 , 6 4}$ & $-0,02$ \\
MBI 04 & 0,17 & $\mathbf{0 , 5 0}$ & 0,18 \\
MBI 10 & 0,05 & 0,11 & $\mathbf{0 , 7 3}$ \\
MBI 11 & 0,13 & 0,09 & $\mathbf{0 , 7 3}$ \\
MBI 15 & 0,05 & 0,05 & $\mathbf{0 , 5 7}$ \\
MBI 05 & 0,27 & 0,15 & $\mathbf{0 , 4 2}$ \\
MBI 22 & 0,27 & 0,04 & $\mathbf{0 , 3 7}$ \\
\hline Autovalor & 6,61 & 2,22 & 1,47 \\
\hline Porcentagem de variância explicada & 30,07 & 10,09 & 6,72 \\
\hline
\end{tabular}

Como se observa na Tabela 1 , os autovalores foram, respectivamente, de 6,61, 2,22 e 1,47 para os fatores 1, 2 e 3 . O total dos fatores explica 46,89\% da variância acumulada das respostas dos sujeitos de nossa amostra ao MBI. O primeiro fator (exaustão emocional), mais forte, explica $30,07 \%$, o segundo (realização profissional) $10,09 \%$ e o terceiro (despersonalização) 6,72 \% da variância.

Os três fatores encontrados através da análise fatorial do MBI em nossa amostra confirmam, tanto em número de itens como semanticamente, as dimensões de burnout propostas por Maslach e 
Jackson (1981): exaustão emocional, baixa realização profissional e despersonalização. Os nove itens que compõem o fator 1 expressam claramente a dimensão de exaustão emocional. O fator 2 , composto de 8 itens, refere-se diretamente aos sentimentos e situações que caracterizam a realização profissional. Já o fator 3 focaliza aspectos indicativos de distanciamento emocional, ou seja, de despersonalização.

Na Tabela 2 se apresentam a média, o desviopadrão e o valor do Alfa de Crombach para cada fator, além das correlações obtidas. A média para a dimensão de Exaustão foi de 2,27 ( $d p=0,67)$, para Realização profissional, 1,60 $(d p=0,52)$ e para Despersonalização, 1,49 (0,48). Ao observarmos os valores de correlação (Pearson) verificamos que são mais fortes as correlações entre exaustão e realização profissional $(0,45, p<0,001)$ e exaustão e despersonalização $(0,44, p<0,001)$, sendo o valor de $r$ de Pearson menor para a correlação entre realização profissional e despersonalização $(0,31, p<0,001)$.

Tabela 2. Médias e desvios padrão, Alfa de Crombach e valores de correlação para as dimensões do MBI.

\begin{tabular}{lcccccc}
\hline & $\mathbf{M}$ & $\mathbf{D p}$ & $\boldsymbol{\alpha}$ & $\mathbf{E E}$ & $\mathbf{B R P}$ & $\mathbf{D E}$ \\
\hline EE & 2,27 & 0,67 & 0,88 & - & $0,45^{* *}$ & $0,44^{* *}$ \\
BRP & 1,60 & 0,52 & 0,82 & $0,45^{* *}$ & - & $0,31^{* *}$ \\
DE & 1,49 & 0,48 & 0,58 & $0,44^{* *}$ & $0,31^{* *}$ & -
\end{tabular}

** $\mathrm{p}<0,001$

EE: Exaustão Emocional; BRP: Baixa Realização Profissional; DE: Despersonalização.

Estes resultados indicam a fidedignidade do MBI em nossa amostra, demonstrando a capacidade deste inventário na medição da síndrome de burnout em professores. Os dois primeiros fatores, exaustão emocional $(\alpha \square 0,88)$ e realização profissional ( $\alpha$ $0,82)$, constituem-se em subescalas com alto índice de consistência interna, podendo constituir-se, estatisticamente, em escalas independentes. $\mathrm{O}$ fator 3 - despersonalização - obteve um coeficiente considerado médio, de 0,58 , próximo ao do reteste realizado pelas autoras do instrumento (Maslach \&Jackson, 1986), isto é, de 0,60. A partir dos resultados obtidos, conclui-se que cada uma das subescalas do Inventário de burnout tem sua confiabilidade interna de moderada a alta.

\section{DISCUSSÃO}

Resultados obtidos nesta amostra confirmam o modelo teórico de burnout no que diz respeito à constituição de três dimensões, como propõe o inventário original americano (Malach \& Jackson, 1981), assim como estudos obtidos em outros países que se propuseram a avaliar a validade de construto deste inventário (Koeske \& Koeske, 1989; Byrne, 1993; Moreno e cols., 1997). Em nossa realidade, resultado similar foi encontrado por Tamayo (1997) em uma amostra de auxiliares de enfermagem e de enfermeiras.

Os três fatores explicam, neste estudo, $46,89 \%$ da variância, resultado bastante semelhante ao encontrado no estudo original americano de Maslach e Jackson (1986), o qual identificou 46,04\% da variância explicada. Koeske e Koeske (1989) encontraram $51 \%$ em seu estudo com trabalhadores sociais americanos, e Gil-Monte e Peiró (1999) identificaram 43,7\% com uma amostra multifuncional de trabalhadores espanhóis

O teste de fidedignidade avaliado pelo Alfa de Cronbach, neste estudo, constatou que as subescalas de exaustão emocional $(0,88)$ e baixa realização profissional no trabalho $(0,82)$ apresentam uma boa consistência interna (alfa >0,70), o que possibilita a criação de um índice a partir da média dos escores atribuídos aos itens pertencentes a estas subescalas. De acordo com Lautert (1995), a subescala de despersonalização possui fidedignidade mais baixa nas diferentes investigações, exceto quando aplicada em norte-americanos, no idioma original em que a mesma foi elaborada. Quanto à fidedignidade da subescala de realização profissional, esta se apresenta mais alta em outros idiomas do que no original, resultado confirmado pelo índice obtido neste estudo (Tabela 3).

Tabela 3. Consistência interna do MBI - Teste $\alpha$ de Cronbach. Comparação transcultural.

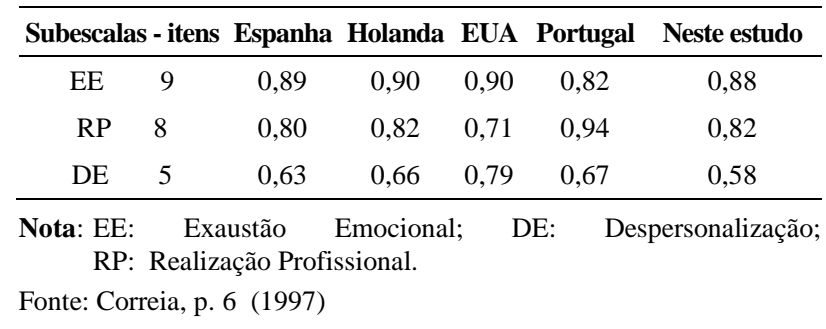

Com relação à subescala de exaustão emocional, os índices obtidos no estudo seguem a tendência de estudos de outros países. Esse aspecto pode evidenciar que esta dimensão é a menos vulnerável a questões culturais, uma vez que o sentimento de desgaste pelo trabalho e as questões que o abordam têm um caráter mais universal, pela estreita relação desta dimensão com o construto de estresse. 
No caso das demais subescalas, considerando-se a síndrome de forma mais ampla, é possível pensar que diferenças culturais tenham influência relevante na maneira como a síndrome de burnout se apresenta em diferentes países, especialmente no que se refere aos indicadores de realização profissional. Embora a escala esteja validada em diferentes realidades, os resultados comparativos demonstram maior consistência interna dessa dimensão nos países europeus e, inclusive, no Brasil. Considerando que o presente estudo foi realizado no Rio Grande do Sul, estado brasileiro com forte imigração européia, acreditamos ser plausível manejar esta hipótese.

Por outro lado, no que tange à dimensão de despersonalização, a validade de construto encontrada neste estudo vai ao encontro das considerações de Koeske e Koeske (1989), que ressaltam a discrepância quantitativa entre o número de itens componentes de cada subescala. Nesta comparação, observamos que a dimensão de despersonalização é medida apenas com cinco itens, o que pode estar influenciando o comportamento do inventário, posto que o construto de despersonalização, potencialmente, está composto de mais aspectos além de "tornar-se mais duro com as pessoas", "preocupar-se por tornar-se mais rígido emocionalmente", "não importar-se pelas pessoas que atende profissionalmente", "sentir-se tratando com as pessoas como se fossem objetos impessoais" e "perceber que os receptores do trabalho culpam os sujeitos por alguns de seus problemas".

Relacionamos aqui, os itens componentes desta dimensão ainda por outros aspectos, que gostaríamos de ressaltar. Primeiramente, retomamos os aspectos culturais. Em algumas culturas, a preocupação por este enrijecimento emocional pode ser relevante e bastante considerada pelos trabalhadores, entretanto, em outras, a simples idéia de que este fator possa estar fazendo-se presente pode representar uma ameaça tanto externa quanto interna. Externamente, seria o caso de sentir-se melindrado frente ao próprio posto de trabalho. Nesse sentido, poderíamos pensar em um efeito de desejabilidade social sobre as respostas a esta dimensão. Internamente, a própria percepção do trabalho como um aspecto ameaçador para os diversos âmbitos de relação do indivíduo pode ser afastada do campo cognitivo no momento de responder ao inventário.

Manejamos esta hipótese considerando, especialmente, os fatores envolvidos em nossa realidade, de uma profissão (docência) valorizada socialmente, porém desvalorizada economicamente, o que faz com que muitos indivíduos utilizem-se da estratégia de evitação cognitiva, na tentativa de dissociar o trabalho (em alguns casos, frustrante) da vida pessoal. Além disso, considerando a docência como uma profissão que permeia e caracteriza de forma muito específica a vida do professor, podemos pensar que as respostas a esta dimensão possam apresentar algum viés.

Conforme Nacarato, Varani e Carvalho (2000), “a atividade do professor é muitas vezes entendida/vivenciada como vocação missionária, negando-se à sua ação uma dimensão crítica da ética e das políticas educacionais" (p. 77). O perfil idealizado de professor funciona, segundo Moura (1997), como uma estratégia defensiva elaborada pelo professor de escolas particulares.

Consideramos, também, em termos semânticos, a possibilidade real de os sujeitos compreenderem os itens referentes a essa dimensão. Possivelmente os aspectos abordados não estejam conectados, de maneira lógica, ao contexto de trabalho. Chamamos atenção especialmente para o item 22, que talvez não seja adequado à realidade de trabalhadores em geral. No caso de professores, os receptores de seu trabalho são os alunos e também seus pais. Estes podem apresentar diversos comportamentos que levem o professor a apresentar indicadores de despersonalização; entretanto, não operam como chefes ou superiores, que atribuiriam ao docente a responsabilidade por seus problemas. Mesmo sendo a realidade da sala de aula um ambiente onde o professor possa ser culpado por baixos índices de desempenho, não podemos afirmar que o pensamento do sujeito se direcione a esta questão.

Após o estudo do MBI em nossa amostra, embora tenhamos encontrado baixo índice de consistência interna em uma de suas dimensões (despersonalização), podemos afirmar que este inventário apresenta uma distribuição fatorial bastante clara, ou seja, que em estudos realizados, em análise fatorial sem delimitação de fatores, as três dimensões se apresentam claramente definidas (Koeske \& Koeske, 1989; Byrne, 1993; Moreno e cols., 1997; Gil-Monte \& Peiró (1999).

As subescalas de exaustão emocional e realização profissional alcançam altos níveis de consistência interna, enquanto despersonalização alcança um índice médio. Neste sentido, nosso estudo apóia as sugestões de Koeske e Koeske (1989), ao analisar a validade de construto do MBI de que as três dimensões poderiam ser revisadas visando alcançar um número-padrão de itens , sugerindo que elas devam possuir 10 itens. Ressalta-se principalmente a necessidade de inclusão de novos itens na dimensão de despersonalização, em função de esta possuir somente cinco questões. 
Como o inventário avalia uma síndrome, é preciso considerar uma série de aspectos que ainda podem ser inseridos como itens no MBI. Entretanto, no atual estado da arte dos estudos sobre burnout, o MBI apresenta-se como uma escala válida e fidedigna nas diferentes realidades onde a síndrome tem sido estudada. Considerando a amostra de professores utilizada no presente estudo, podemos afirmar que a versão brasileira do $\mathrm{MBI}$ apresenta os requisitos necessários em termos de consistência interna e validade fatorial para ser amplamente utilizada na avaliação da síndrome de burnout em nossa realidade.

\section{REFERÊNCIAS}

Bisquerra-Alzina, R. (1989). Introducción conceptual al análisis multivariable. Barcelona: PPU

Byrne, B. M. (1993). The Maslach Burnout Inventory: Testing for factorial validity and invariance across elementary, intermediate and secondary teachers. Journal of Occupational and Organizational Psychology, 66(3), 197-213.

Carlotto, M. S. (2002). Síndrome de Burnout e satisfação no trabalho: um estudo com professores universitários. Em A. M. T. Benevides-Pereira (Org), Burnout: quando o trabalho ameaça o bem estar do trabalhador (pp. 187-212). São Paulo: Casa do Psicólogo.

Carlotto, M.S. (2003). Síndrome de burnout e gênero em docentes de instituições particulares de ensino. Revista de Psicologia da Universidade do Contestado, 1 (1) Disponível em: www.nead.uncnet.br/revista/psicologia. (Acessado em 24/12/2003)

Cordes C. L., \& Dougherty, T. W. (1993). A review and integration of research on job burnout. Academy of Management Review, 18 (4) 632- 636.

Farber, B. A. (1991). Crisis in education. Stress and burnout in the american teacher. São Francisco: Jossey-Bass Inc.

França, H. H. (1987). A síndrome de burnout. Revista Brasileira de Medicina, 44(8) 197-199.

Freudenberger, H. J. (1974). Staff burnout. Journal of Social Issues, 30, 159-165.

Gil-Monte, P. R., \& Peiró, J. M. (1997). Desgaste psíquico en el trabajo: El síndrome de quemarse. Madrid: Sínteses.

Gil-Monte, P. R., \& Peiró, J. M. (1999). Validez factorial del Maslach Burnout Inventory en una muestra multiocupacional. Psicothema, 11(3), 679-689.

Hutz, C. S. \& Spink, M. J. (1996). Orientações éticas para psicólogos envolvidos em pesquisas com seres humanos. Disponível em: http://www.psicologia.urgrs.etica2.htm. (Acessado em 09/03/2002)

Koeske, G. F., \& Koeske, R. D. (1989). Construct validity of the Maslach Burnout Inventory: a critical review and reconceptualization. The Journal of Applied Behavioral Science 25(2) 131-144.
Lautert, L. (1995). O desgaste profissional do enfermeiro. Tese de doutorado Não-Publicada, Programa de Pós Graduação em Psicologia, Universidade Pontificia de Salamanca - Facultad de Psicologia, Salamanca.

Maslach, C. (1993). Burnout: A multidimensional perspective. Em W.B.Schaufeli, C. Maslach \& T. Marek (Eds.), Professional burnout: Recent developments in theory and research (pp.19-32). New York: Taylor \& Francis.

Maslach, C., \& Goldberg, J. (1998). Prevention of burnout: News perspectives. Applied \& Preventive Psychology, 7, 63-74.

Maslach, C., \& Jackson, S. E. (1981). The measurement of experienced burnout. Journal of Ocuppational Behavior, 2, 99113.

Maslach, C., \& Jackson, S. E. (1986) (2nd ed). Maslach Burnout Inventory. Palo Alto, CA: Consulting Psychologist Press.

Maslach, C., S.E., Jackson \& Leiter, M. P. (1996, $3^{\text {rd }}$ ed.). The Maslach Burnout Inventory - Test manual. Palo Alto, CA. Consulting Psycologist Press.

Maslach, C., \& Leiter, M. P. (1997). The truth about burnout: How organization cause, personal stress and what to do about It. San Francisco: Jossey-Bass.

Maslach, C., Schaufeli, W. B., \& Leiter, M. P. (2001). Job burnout. Annual Review Psychology, 52, 397-422.

Moreno, B. J., Bustos, R. R., Matallana, A. A., \& Mirrales, C. T. (1997). La evaluacion del burnout. Problemas y alternativas. El CBB como avaliação dos elementos do processo. Revista de Psicología del Trabajo, 13(2) 185-207.

Moura, E. P. G. (1997). Saúde mental e trabalho. Esgotamento profissional em professores da Rede de Ensino Particular de Pelotas - RS. Dissertação de Mestrado Não-Publicada, Programa de Pós-graduação em Psicologia, Pontifícia Universidade Católica do Rio Grande do Sul, Porto Alegre.

Nacarato, A.M.; Varani, A. \& Carvalho,V. (2000). O cotidiano do trabalho docente: palco, bastidores e trabalho invisível...abrindo as cortinas. Em C. M. G Geraldi, D. Fiorentina \& E. M. de A. Pereira (Orgs.), Cartografias do trabalho docente (pp.45-79). Campinas: Mercado de Letras.

Perlman, B., \& Hartman A. E. (1982). Burnout: Sumary and future research. Human Relations, 35(4), 283-305.

Schaufeli, W. B., Maslach, C., \& Marek, T. (1993). Professional burnout. Recent developments in theory and research. New York: Taylor \& Francis.

Tamayo, R. M. (1997). Relação entre a síndrome de burnout e os valores organizacionais no pessoal de enfermagem de dois hospitais públicos. Dissertação de Mestrado Não-Publicada. Instituto de Psicologia, Universidade de Brasília.

Recebido em 30/01/2004 Aceito em 31/08/2004

Endereço para correspondência: Mary Sandra Carlotto: Av. Mauá, 645, apto. 504, Centro, CEP 93110-320, São Leopoldo-RS. Email: mscarlotto@ superig.com.br 


\section{ERRATA}

Psicologia em Estudo, Maringá, v. 9, n. 3, p. 499-505, set./dez. 2004

Carlotto, M. S., Câmara, S. G. Análise fatorial do Maslach Burnout Inventory (MBI) em uma amostra de professores de instituições particulares. Psicologia em Estudo, 9(3), 499-505. http://www.scielo.br/pdf/pe/v9n3/v9n3a17.pdf.

A Tabela 1 foi substituda pela atualmente publicada em atenção ao pedido do proprietário do material em cumprimento a lei de direitos autorais do The Digital Millennium Copyright Act (DMCA). 